\title{
On the blow-up criterion of strong solutions for the MHD equations with the Hall and ion-slip effects in $\mathbb{R}^{3}$
}

\author{
Sadek Gala and Maria Alessandra Ragusa
}

\begin{abstract}
In this paper, we establish a blow-up criterion of strong solutions to the 3D incompressible magnetohydrodynamics equations including two nonlinear extra terms: the Hall term (quadratic with respect to the magnetic field) and the ion-slip term (cubic with respect to the magnetic field). This is an improvement of the recent results given by Fan et al. ( $\mathrm{Z}$ Angew Math Phys, 2015).
\end{abstract}

Mathematics Subject Classification. 35Q35 - 35B65 - 76D05.

Keywords. MHD equations - Besov space $\cdot$ Multiplier space $\cdot$ Blow-up criterion · Strong solution.

\section{Introduction}

We are interested in the regularity criterion of the following incompressible magnetohydrodynamics equations with the Hall and ion-slip effects in $\mathbb{R}^{3}$ :

$$
\left\{\begin{array}{c}
\partial_{t} u+(u \cdot \nabla) u-(\nabla \times B) \times B-\Delta u+\nabla \pi=0, \\
\partial_{t} B+(u \cdot \nabla) B-(B \cdot \nabla) u+\nabla \times((\nabla \times B) \times B)-\Delta B=\nabla \times[B \times(B \times(\nabla \times B)], \\
\nabla \cdot u=\nabla \cdot B=0, \\
(u, B)(x, 0)=\left(u_{0}(x), B_{0}(x)\right),
\end{array}\right.
$$

where $x \in \mathbb{R}^{3}$ and $t>0$. Here $u=u(x, t) \in \mathbb{R}^{3}, B=B(x, t) \in \mathbb{R}^{3}$ and $\pi=\pi(x, t)$ are nondimensional quantities corresponding to the flow velocity, the magnetic field and the pressure at the point $(x, t)$, while $u_{0}(x)$ and $B_{0}(x)$ are the given initial velocity and initial magnetic field with $\nabla \cdot u_{0}=0$ and $\nabla \cdot B_{0}=0$, respectively.

Magnetohydrodynamics (MHD) is a fluid theory that describes plasma physics by treating the plasma as a fluid of charged particles. Hence, the equations that describe the plasma form a nonlinear system that couples Navier-Stokes equations with Maxwell's equations. This model describes some important physical phenomena. Comparing with the usual viscous incompressible MHD equations, the system (1.1) contains the extra term $\nabla \times((\nabla \times B) \times B)$ which is the so-called Hall term and $\nabla \times[((\nabla \times B) \times B) \times B]$ the ion-slip effect.

The above system has been studied a lot by physicists and mathematicians because of its physical importance, complexity, rich phenomena and mathematical challenges; see $[1-4,6]$ and the references cited therein.

The mathematical study of the above system was initiated by Mulone and Solonnikov [19], who proved the small data global existence of strong solutions in a bounded domain. Refer to [19-21]. Recently, Chae and Lee [2] established an existence result on strong solutions and proved the following regularity criteria

$$
u \in L^{\frac{2 q}{q-3}}\left(0, T ; L^{q}\left(\mathbb{R}^{3}\right)\right) \quad \text { and } \quad \nabla B \in L^{\frac{2 s}{s-3}}\left(0, T ; L^{s}\left(\mathbb{R}^{3}\right)\right) \text { with } 3<q, s \leq \infty,
$$


or

$$
u \in L^{2}\left(0, T ; \mathrm{BMO}\left(\mathbb{R}^{3}\right)\right) \quad \text { and } \quad \nabla B \in L^{2}\left(0, T ; \mathrm{BMO}\left(\mathbb{R}^{3}\right)\right) .
$$

Here BMO is the space of functions of bounded mean oscillations.

Very recently, the local well posedness of the strong solution to the incompressible magnetohydrodynamic equations with the Hall and ion-slip effects was established for the whole space $\mathbb{R}^{3}$ by Fan et al. [12] and they proved that if

$$
\pi \in L^{\frac{2 q}{2 q-3}}\left(0, T ; L^{q}\left(\mathbb{R}^{3}\right)\right) \quad \text { with } 3<q \leq \infty
$$

and

$$
B \in L^{\infty}\left(0, T ; L^{\infty}\left(\mathbb{R}^{3}\right)\right) \text { and } \nabla B \in L^{\frac{2 q}{q-3}}\left(0, T ; L^{q}\left(\mathbb{R}^{3}\right)\right) \text { with } 3<q \leq \infty,
$$

then the solution $(u(t), B(t))$ can be extended beyond $t=T$.

In this paper, we extend the results of [12] to the critical Besov space. We prove a blow-up criterion for local strong solutions in terms of the critical Besov space $\dot{B}_{\infty ; \infty}^{-1}$ and multiplier spaces.

\section{Multiplier and Besov spaces}

Before stating our results precisely, we first recall the definition of the homogeneous Besov space with negative indices $\dot{B}_{\infty, \infty}^{-\alpha}$ in $\mathbb{R}^{3}$ and the homogeneous Sobolev space $\dot{H}_{q}^{\alpha}$ of exponent $\alpha>0$. It is known [24] that $f \in \mathcal{S}^{\prime}\left(\mathbb{R}^{3}\right)$ belongs to $\dot{B}_{\infty, \infty}^{-\alpha}\left(\mathbb{R}^{3}\right)$ if and only if the heat semigroup $\mathrm{e}^{t \Delta} f \in L^{\infty}\left(\mathbb{R}^{3}\right)$ for all $t>0$ and $t^{\frac{\alpha}{2}}\left\|\mathrm{e}^{t \Delta} f\right\|_{\infty} \in L^{\infty}(0, \infty)$. The norm of $\dot{B}_{\infty, \infty}^{-\alpha}$ is defined, up to equivalence, by

$$
\|f\|_{\dot{B}_{\infty, \infty}^{-\alpha}}=\sup _{t>0}\left(t^{\frac{\alpha}{2}}\left\|\mathrm{e}^{t \Delta} f\right\|_{\infty}\right) \text {. }
$$

We introduce now the homogeneous Sobolev space $\dot{H}_{q}^{s}\left(\mathbb{R}^{3}\right)$, which is defined as the set of functions $f \in L^{r}\left(\mathbb{R}^{3}\right), \frac{1}{r}=\frac{1}{q}-\frac{s}{3}$ such that $(-\Delta)^{\frac{s}{2}} f \in L^{q}\left(\mathbb{R}^{3}\right)$. This space is endowed with the norm

$$
\|f\|_{\dot{H}_{q}^{s}}=\left\|(-\Delta)^{\frac{s}{2}} f\right\|_{L^{q}}
$$

and when $q=2$, we just let $\dot{H}_{2}^{s}\left(\mathbb{R}^{3}\right)=\dot{H}^{s}\left(\mathbb{R}^{3}\right)$. Recall that

$$
\|f\|_{\dot{H}_{q}^{s}} \leq\|f\|_{H_{q}^{s}}, \quad s \geq 0, \quad f \in H_{q}^{s}\left(\mathbb{R}^{3}\right) .
$$

Here $H_{q}^{s}\left(\mathbb{R}^{3}\right)$ is the standard inhomogeneous Sobolev space. We introduce the following two lemmas.

Lemma 2.1. Let $0 \leq \alpha<\frac{3}{2}$. Then the embedding

$$
\dot{H}^{\frac{3}{2}-\alpha}\left(\mathbb{R}^{3}\right) \subset L^{\frac{3}{\alpha}}\left(\mathbb{R}^{3}\right) \subset \dot{B}_{\infty, \infty}^{-\alpha}\left(\mathbb{R}^{3}\right)
$$

holds.

Lemma 2.2. Let $1<p<q<\infty$ and let $s=\alpha\left(\frac{q}{p}-1\right)>0$. Then there exists a constant depending only on $\alpha, p$ and $q$ such that the estimate

$$
\|f\|_{L^{q}} \leq C\left\|(-\Delta)^{\frac{s}{2}} f\right\|_{p}^{\frac{p}{q}}\|f\|_{\dot{B}_{\infty, \infty}^{-\alpha}}^{1-\frac{p}{q}}
$$

holds for all $f \in \dot{H}_{p}^{s}\left(\mathbb{R}^{3}\right) \cap \dot{B}_{\infty, \infty}^{-\alpha}\left(\mathbb{R}^{3}\right)$.

This result is due to Meyer-Gerard-Oru [18], and the proof is based on the Littlewood-Paley decomposition, please refer to [18] for the details.

Next, let us define the function spaces in which the blow-up criterion of strong solution $(u, B, \pi)$ is going to be established. 
Definition 2.3. $[16,26]$ For $0 \leq r<\frac{3}{2}$, the space $\dot{X}_{r}$ is defined as the space of $f(x) \in L_{\text {loc }}^{2}\left(\mathbb{R}^{3}\right)$ such that

$$
\|f\|_{\dot{X}_{r}}=\sup _{\|g\|_{\dot{H}^{r}} \leq 1}\|f g\|_{L^{2}}<\infty
$$

where we denote by $\dot{H}^{r}\left(\mathbb{R}^{3}\right)$ the completion of the space $\mathcal{D}\left(\mathbb{R}^{3}\right)$ with respect to the norm $\|u\|_{\dot{H}^{r}}=$ $\left\|(-\Delta)^{\frac{r}{2}} u\right\|_{L^{2}}$.

We have the homogeneity properties: $\forall x_{0} \in \mathbb{R}^{3}$

$$
\begin{aligned}
\left\|f\left(.+x_{0}\right)\right\|_{\dot{X}_{r}} & =\|f\|_{\dot{X}_{r}} \\
\|f(\lambda .)\|_{\dot{X}_{r}} & =\frac{1}{\lambda^{r}}\|f\|_{\dot{X}_{r}}, \quad \lambda>0 .
\end{aligned}
$$

Lemma 2.4. Let $0 \leq r<\frac{3}{2}$. Then

$$
L^{\frac{3}{r}}\left(\mathbb{R}^{3}\right) \subset \dot{X}_{r}\left(\mathbb{R}^{3}\right)
$$

holds.

Proof. Let $f \in L^{\frac{3}{r}}\left(\mathbb{R}^{3}\right)$. By using the following well-known Sobolev embedding

$$
\dot{H}^{r}\left(\mathbb{R}^{3}\right) \subset L^{q}\left(\mathbb{R}^{3}\right)
$$

with $\frac{1}{q}=\frac{1}{2}-\frac{r}{3}$, we have

$$
\|f g\|_{L^{2}} \leq\|f\|_{L^{\frac{3}{r}}}\|g\|_{L^{q}} \leq C\|f\|_{L^{\frac{3}{r}}}\|g\|_{\dot{H}^{r}}
$$

Then, it follows that

$$
\|f\|_{\dot{X}_{r}}=\sup _{\|g\|_{\dot{H}^{r}} \leq 1}\|f g\|_{L^{2}} \leq C\|f\|_{L^{\frac{3}{r}}} .
$$

Remark 2.1. We note from Proposition 2.5 in [16] that $\dot{X}_{r} \subset \dot{B}_{\infty ; \infty}^{-r}$ for all $0 \leq r<\frac{3}{2}$.

By Banach fixed point theorem and energy estimates, it is easy to prove the following well posedness of local strong solutions to the problem (1.1), and hence we omit the details here (see for e.g., [12]).

Theorem 2.5. Let $\left(u_{0}, B_{0}\right) \in H^{2}\left(\mathbb{R}^{3}\right) \times H^{2}\left(\mathbb{R}^{3}\right)$ with $\nabla \cdot u_{0}=\nabla \cdot B_{0}=0$. There exists a positive time $T$ such that the problem $(1.1)$ has a unique strong solution $(u, B)$ in $(0, T)$ such that

$$
\begin{aligned}
(u, B) & \in L^{\infty}\left(0, T ; H^{2}\left(\mathbb{R}^{3}\right)\right) \cap L^{2}\left(0, T ; H^{3}\left(\mathbb{R}^{3}\right)\right), \\
\left(\partial_{t} u, \partial_{t} B\right) & \in L^{\infty}\left(0, T ; L^{2}\left(\mathbb{R}^{3}\right)\right) \cap L^{2}\left(0, T ; H^{1}\left(\mathbb{R}^{3}\right)\right) .
\end{aligned}
$$

First our main result reads as follows.

Theorem 2.6. Let the initial data $\left(u_{0}, B_{0}\right) \in H^{2}\left(\mathbb{R}^{3}\right)$ with $\nabla \cdot u_{0}=\nabla \cdot B_{0}=0$. Assume that $(u, B)$ is a local strong solution to Hall-MHD equations on some interval $[0, T]$ with $0<T<\infty$ constructed in Theorem 2.5. If $(u, B, \pi)$ satisfies the following condition

$$
(u, \nabla B) \in L^{\frac{2}{1-r}}\left(0, T ; \dot{X}_{r}\left(\mathbb{R}^{3}\right)\right) \text { with } 0 \leq r<1
$$

and

$$
B \in L^{\infty}\left(0, T ; L^{\infty}\left(\mathbb{R}^{3}\right)\right),
$$

then the solution $(u, B)$ can be extended beyond $T$. 
Next, we consider an improvement of Theorem 2.6 for the case $r=1$ by using critical Besov space $\dot{B}_{\infty ; \infty}^{-1}$, in which $\dot{X}_{1}\left(\mathbb{R}^{3}\right)$ is embedded.

Theorem 2.7. Let the initial data $\left(u_{0}, B_{0}\right) \in H^{2}\left(\mathbb{R}^{3}\right)$ with $\nabla \cdot u_{0}=\nabla \cdot B_{0}=0$. Assume that $(u, B)$ is a local strong solution to Hall-MHD equations on some interval $[0, T]$ with $0<T<\infty$ constructed in Theorem 2.5. If $(u, B, \pi)$ satisfies the following condition

$$
\pi \in L^{2}\left(0, T ; \dot{B}_{\infty ; \infty}^{-1}\left(\mathbb{R}^{3}\right)\right)
$$

and

$$
B \in L^{\infty}\left(0, T ; L^{\infty}\left(\mathbb{R}^{3}\right)\right) \quad \text { and } \quad \nabla B \in L^{\frac{2}{1-r}}\left(0, T ; \dot{X}_{r}\left(\mathbb{R}^{3}\right)\right) \text { with } 0 \leq r<1
$$

then the solution $(u, B)$ can be extended beyond $T$.

Remark 2.2. Since the multiplier space $\dot{X}_{r}\left(\mathbb{R}^{3}\right)$ with $0 \leq r \leq 1$ is wider than the Lebesgue space $L^{\frac{3}{r}}\left(\mathbb{R}^{3}\right)$, hence our result is an improvement of the recent result obtained by Chae-Lee [2] and Fan et al. [12].

Remark 2.3. When $r=0$, we notice that there holds $\dot{X}_{0}\left(\mathbb{R}^{3}\right) \cong \operatorname{BMO}\left(\mathbb{R}^{3}\right)$, where BMO denotes the space of bounded mean oscillations. Hence, our result in Theorem 2.6 gives that the condition

$$
(u, \nabla B) \in L^{2}\left(0, T ; \operatorname{BMO}\left(\mathbb{R}^{3}\right)\right)
$$

still implies the local strong solution $(u, B)$ is regular on $\mathbb{R}^{3} \times(0, T]$.

Remark 2.4. In the sequel, we will use the following inequality for all $1<q<\infty$

$$
\|\nabla \pi\|_{L^{q}} \leq C\left(\|u \cdot \nabla u\|_{L^{q}}+\|B \times(\nabla \times B)\|_{L^{q}}\right)
$$

\section{Proof of Theorem 2.6}

We are now in a position to the proof of our first result.

Proof. We want to establish an a priori estimate for the smooth solution.

First, multiplying $(1.1)_{1}$ by $u$, after integration by parts and taking the divergence-free property into account, we have

$$
\frac{1}{2} \frac{\mathrm{d}}{\mathrm{d} t} \int_{\mathbb{R}^{3}}|u|^{2} \mathrm{~d} x+\|\nabla u\|_{L^{2}}^{2}=\int_{\mathbb{R}^{3}}((\nabla \times B) \times B) \cdot u \mathrm{~d} x=-\int_{\mathbb{R}^{3}}(u \times B) \cdot \nabla \times B \mathrm{~d} x .
$$

Similarly, multiplying $(1.1)_{2}$ by $B$, and integrating over $\mathbb{R}^{3}$, we have

$$
\frac{1}{2} \frac{\mathrm{d}}{\mathrm{d} t} \int_{\mathbb{R}^{3}}|B|^{2} \mathrm{~d} x+\|\nabla B\|_{L^{2}}^{2}+\|B \times(\nabla \times B)\|_{L^{2}}^{2}=\int_{\mathbb{R}^{3}}(u \times B) \cdot \nabla \times B \mathrm{~d} x .
$$

Combining (3.1) and (3.2), we have the well-known energy equality

$$
\frac{1}{2} \frac{\mathrm{d}}{\mathrm{d} t} \int_{\mathbb{R}^{3}}\left(|u|^{2}+|B|^{2}\right) \mathrm{d} x+\|\nabla u\|_{L^{2}}^{2}+\|\nabla B\|_{L^{2}}^{2}+\|(\nabla \times B) \times B\|_{L^{2}}^{2}=0 .
$$

Integrating the above equality over time interval $[0, t]$, we obtain the energy equality and this proves that

$$
(u, B) \in L^{\infty}\left(0, T ; L^{2}\left(\mathbb{R}^{3}\right)\right) \cap L^{2}\left(0, T ; H^{1}\left(\mathbb{R}^{3}\right)\right) .
$$

Take operator $\Delta$ on Eq. $(1.1)_{1}$ and take scalar product of them with $\Delta u$, we obtain 


$$
\begin{aligned}
& \frac{1}{2} \frac{\mathrm{d}}{\mathrm{d} t} \int_{\mathbb{R}^{3}}|\Delta u|^{2} \mathrm{~d} x+\int_{\mathbb{R}^{3}}|\nabla \Delta u|^{2} \mathrm{~d} x \\
&\left.\quad=-\int_{\mathbb{R}^{3}} \Delta(u \cdot \nabla u) \cdot \Delta u d x+\int_{\mathbb{R}^{3}} \Delta((\nabla \times B) \times B)\right) \cdot \Delta u \mathrm{~d} x=J_{1}+J_{2} .
\end{aligned}
$$

Now let us remind the following well-known interpolation inequality: if $0 \leq r \leq 1$, then

$$
\|f\|_{\dot{H}^{r}} \leq C\|f\|_{L^{2}}^{1-r}\|\nabla f\|_{L^{2}}^{r} .
$$

Using this inequality together with Young's inequality, we obtain

$$
\begin{aligned}
J_{1} & =-\sum_{i=1}^{3} \int_{\mathbb{R}^{3}} \Delta u_{i} \partial_{i} u \cdot \Delta u \mathrm{~d} x-2 \sum_{i, j=1}^{3} \int_{\mathbb{R}^{3}} \partial_{j} u_{i} \partial_{i} \partial_{j} u \cdot \Delta u \mathrm{~d} x \\
& =\sum_{i=1}^{3} \int_{\mathbb{R}^{3}} \Delta u_{i} u \cdot \partial_{i} \Delta u \mathrm{~d} x+2 \sum_{i, j=1}^{3} \int_{\mathbb{R}^{3}} u_{i} \partial_{j}\left(\partial_{i} \partial_{j} u \cdot \Delta u\right) \mathrm{d} x \\
& \leq C\|u \cdot \Delta u\|_{L^{2}}\|\nabla \Delta u\|_{L^{2}} \\
& \leq C\|u\|_{\dot{X}_{r}}\|\Delta u\|_{\dot{H}^{r}}\|\nabla \Delta u\|_{L^{2}} \\
& \leq \frac{1}{4}\|\nabla \Delta u\|_{L^{2}}^{2}+C\|u\|_{\dot{X}_{r}}^{\frac{2}{1-r}}\|\Delta u\|_{L^{2}}^{2} .
\end{aligned}
$$

In the following calculations we will use the following Gagliardo-Nirenberg inequality

$$
\|\nabla f\|_{L^{2 q}}^{2} \leq C\|f\|_{L^{\infty}}\|\Delta f\|_{L^{q}} \text { for } \quad 1<q<\infty .
$$

To estimate $J_{2}$, we apply interpolation inequality and Young's inequality,

$$
\begin{aligned}
J_{2} & \leq C \int_{\mathbb{R}^{3}}\left(|\nabla B|^{2}+|B||\Delta B|\right)|\nabla \Delta u| \mathrm{d} x \\
& \leq C\left(\|\nabla B\|_{L^{4}}^{2}+\|B\|_{L^{\infty}}\|\Delta B\|_{L^{2}}\right)\|\nabla \Delta u\|_{L^{2}} \\
& \leq \frac{1}{4}\|\nabla \Delta u\|_{L^{2}}^{2}+C\|B\|_{L^{\infty}}^{2}\|\Delta B\|_{L^{2}}^{2} .
\end{aligned}
$$

Applying $\Delta$ to $(1.1)_{2}$, then multiplying it by $\Delta B$, after integration by parts, we find that,

$$
\begin{aligned}
\frac{1}{2} & \frac{\mathrm{d}}{\mathrm{d} t} \int_{\mathbb{R}^{3}}|\Delta B|^{2} \mathrm{~d} x+\int_{\mathbb{R}^{3}}|\nabla \Delta B|^{2} \mathrm{~d} x+\int_{\mathbb{R}^{3}}|B \times(\nabla \times \Delta B)|^{2} \mathrm{~d} x \\
= & \int_{\mathbb{R}^{3}}[\nabla \times \Delta(u \times B)] \cdot \Delta B \mathrm{~d} x-\int_{\mathbb{R}^{3}} \Delta((\nabla \times B) \times B) \cdot(\nabla \times \Delta B) \mathrm{d} x \\
& \left.+\int_{\mathbb{R}^{3}}\{\Delta[(\nabla \times B) \times B) \times B] \cdot(\nabla \times \Delta B)+|B \times(\nabla \times \Delta B)|^{2}\right\} \mathrm{d} x \\
= & I_{1}+I_{2}+I_{3} .
\end{aligned}
$$

From the calculus inequality, interpolation inequality and Young's inequality, we bound $I_{1}$ as follows:

$$
\begin{aligned}
I_{1} & =\int_{\mathbb{R}^{3}}[\Delta(u \times B)] \cdot(\nabla \times \Delta B) \mathrm{d} x \\
& =\int_{\mathbb{R}^{3}}\left[\Delta u \times B+u \times \Delta B+2 \sum_{i=1}^{3} \partial_{i} u \times \partial_{i} B\right] \cdot(\nabla \times \Delta B) \mathrm{d} x
\end{aligned}
$$




$$
\begin{aligned}
& \leq\left(\|\Delta u\|_{L^{2}}\|B\|_{L^{\infty}}+\|u \cdot \Delta B\|_{L^{2}}+2\|\nabla u \cdot \nabla B\|_{L^{2}}\right)\|\nabla \Delta B\|_{L^{2}} \\
& \leq \frac{1}{16}\|\nabla \Delta B\|_{L^{2}}^{2}+C\left(\|\Delta u\|_{L^{2}}^{2}\|B\|_{L^{\infty}}^{2}+\|u\|_{\dot{X}_{r}}^{2}\|\Delta B\|_{\dot{H}^{r}}^{2}+4\|\nabla B\|_{\dot{X}_{r}}^{2}\|\nabla u\|_{\dot{H}^{r}}^{2}\right) \\
& \leq \frac{1}{8}\|\nabla \Delta B\|_{L^{2}}^{2}+C\|\Delta u\|_{L^{2}}^{2}\|B\|_{L^{\infty}}^{2}+C\|u\|_{\dot{X}_{r}}^{\frac{2}{1-r}}\|\Delta B\|_{L^{2}}^{2}+C\|\nabla B\|_{\dot{X}_{r}}^{\frac{2}{1-r}}\|\nabla u\|_{L^{2}}^{2}+C\|\Delta u\|_{L^{2}}^{2} \\
& \leq \frac{1}{8}\|\nabla \Delta B\|_{L^{2}}^{2}+C\|u\|_{\dot{X}_{r}}^{\frac{2}{1-r}}\|\Delta B\|_{L^{2}}^{2}+C\|\nabla B\|_{\dot{X}_{r}}^{\frac{2}{1-r}}\|\nabla u\|_{L^{2}}^{2}+C\|\Delta u\|_{L^{2}}^{2}\left(1+\|B\|_{L^{\infty}}^{2}\right) .
\end{aligned}
$$

Similarly, from the calculus inequality, interpolation inequality and Young's inequality, we bound $I_{2}$ as follows:

$$
\begin{aligned}
I_{2} & \left.=-\int_{\mathbb{R}^{3}}\left[(\nabla \times B) \times \Delta B+2 \sum_{i=1}^{3} \partial_{i}(\nabla \times B) \times \partial_{i} B\right)\right] \cdot(\nabla \times \Delta B) \mathrm{d} x \\
& \leq C\|\nabla B\|_{\dot{X}_{r}}\|\Delta B\|_{\dot{H}^{r}}\|\nabla \Delta B\|_{L^{2}} \\
& \leq \frac{1}{8}\|\nabla \Delta B\|_{L^{2}}^{2}+C\|\nabla B\|_{\dot{X}_{r}}^{\frac{2}{1-r}}\|\Delta B\|_{L^{2}}^{2} .
\end{aligned}
$$

By integration by parts, $I_{3}$ can be rewritten as:

$$
\begin{aligned}
I_{3}= & \int_{\mathbb{R}^{3}}\left[\sum_{i=1}^{3} \partial_{i}\left[\left(\partial_{i}(\nabla \times B) \times B\right) \times B+\left((\nabla \times B) \times \partial_{i} B\right) \times B+((\nabla \times B) \times B) \times \partial_{i} B\right] \mathrm{d} x\right. \\
& +\int_{\mathbb{R}^{3}}|B \times \Delta(\nabla \times B)|^{2} \mathrm{~d} x \\
= & \sum_{i=1}^{3} \int_{\mathbb{R}^{3}}\left[2\left(\partial_{i}(\nabla \times B) \times \partial_{i} B\right) \times B+2\left(\partial_{i}(\nabla \times B) \times B\right) \times \partial_{i} B+\left((\nabla \times B) \times \partial_{i}^{2} B\right) \times B\right. \\
& \left.+((\nabla \times B) \times B) \times \partial_{i}^{2} B\right] \cdot(\nabla \times \Delta B) \mathrm{d} x+2 \sum_{i=1}^{3} \int_{\mathbb{R}^{3}}\left[\left((\nabla \times B) \times \partial_{i} B\right) \times \partial_{i} B\right] \cdot(\nabla \times \Delta B) \mathrm{d} x \\
= & I_{3,1}+I_{3,2} .
\end{aligned}
$$

For $I_{3,1}$, using Young's inequality and the interpolation inequality, we obtain

$$
\begin{aligned}
I_{3,1} & \leq C\|B\|_{L^{\infty}}\|\nabla B\|_{\dot{X}_{r}}\|\Delta B\|_{\dot{H}^{r}}\|\nabla \Delta B\|_{L^{2}} \\
& \leq \frac{1}{8}\|\nabla \Delta B\|_{L^{2}}^{2}+C\|B\|_{L^{\infty}}^{\frac{2}{1-r}}\|\nabla B\|_{\dot{X}_{r}}^{\frac{2}{1-r}}\|\Delta B\|_{L^{2}}^{2} .
\end{aligned}
$$

For the second term on the right of $I_{3}$, by the simple vector identity and Hölder inequality, $I_{3,2}$ is bounded by

$$
\begin{aligned}
I_{3,2} & =2 \sum_{i=1}^{3} \int_{\mathbb{R}^{3}} \nabla \times\left[\left((\nabla \times B) \times \partial_{i} B\right) \times \partial_{i} B\right] \cdot \Delta B \mathrm{~d} x \\
& \leq \frac{1}{8}\|\nabla \Delta B\|_{L^{2}}^{2}+C\|\nabla B\|_{\dot{X}_{r}}^{\frac{2}{1-r}}\|\Delta B\|_{L^{2}}^{2} .
\end{aligned}
$$

Inserting the estimates above in (3.3)-(3.4), we obtain

$$
\begin{aligned}
& \frac{1}{2} \frac{\mathrm{d}}{\mathrm{d} t} \int_{\mathbb{R}^{3}}\left(|\Delta u|^{2}+|\Delta B|^{2}\right) \mathrm{d} x+\int_{\mathbb{R}^{3}}\left(|\nabla \Delta u|^{2}+|\nabla \Delta B|^{2}\right) \mathrm{d} x+\int_{\mathbb{R}^{3}}|B \times(\nabla \times \Delta B)|^{2} \mathrm{~d} x \\
& \quad \leq \frac{1}{2}\|\nabla \Delta u\|_{L^{2}}^{2}+C\left(\|u\|_{\dot{X}_{r}}^{\frac{2}{1-r}}+\|B\|_{L^{\infty}}^{2}\right)\left(\|\Delta u\|_{L^{2}}^{2}+\|\Delta B\|_{L^{2}}^{2}\right)
\end{aligned}
$$




$$
+\frac{1}{2}\|\nabla \Delta B\|_{L^{2}}^{2}+C\|\nabla B\|_{\dot{X}_{r}}^{\frac{2}{1-r}}\left(\|\Delta u\|_{L^{2}}^{2}+\|\Delta B\|_{L^{2}}^{2}+\|B\|_{L^{\infty}}^{\frac{2}{1-r}}\|\Delta B\|_{L^{2}}^{2}\right) .
$$

Consequently, using the Gronwall's inequality, one has

$$
(u, B) \in L^{\infty}\left(0, T ; H^{2}\left(\mathbb{R}^{3}\right)\right) \cap L^{2}\left(0, T ; H^{3}\left(\mathbb{R}^{3}\right)\right) .
$$

This completes the proof of Theorem 2.6.

\section{Proof of Theorem 2.7}

In this section, we shall give the proof of theorem 2.7.

Proof. Testing $(1.1)_{2}$ by $|u|^{2} u$, taking the divergence-free property into account and using the Hölder inequality, we get

$$
\begin{aligned}
& \frac{1}{4} \frac{\mathrm{d}}{\mathrm{d} t} \int_{\mathbb{R}^{3}}|u|^{4} \mathrm{~d} x+\frac{1}{2}\|u \cdot \nabla u\|_{L^{2}}^{2}+\frac{1}{2}\left\|\nabla|u|^{2}\right\|_{L^{2}}^{2} \\
& \quad=\int_{\mathbb{R}^{3}}\left(B \cdot \nabla B-\frac{1}{2} \nabla|B|^{2}\right) \cdot|u|^{2} u \mathrm{~d} x-\int_{\mathbb{R}^{3}} u|u|^{2} \cdot \nabla \pi \mathrm{d} x \\
& \quad=\int_{\mathbb{R}^{3}} \pi \nabla \cdot\left(u|u|^{2}\right) \mathrm{d} x+\frac{1}{2} \int_{\mathbb{R}^{3}} \nabla \cdot\left(|u|^{2} u\right) \cdot|B|^{2} \mathrm{~d} x-\sum_{i=1}^{3} \int_{\mathbb{R}^{3}} B_{i} B \partial_{i}\left(|u|^{2} u\right) \mathrm{d} x \\
& \leq C \int_{\mathbb{R}^{3}}|\pi||u|^{2}|\nabla u| \mathrm{d} x+C \int_{\mathbb{R}^{3}}|B|^{2}|u||u \cdot \nabla u| \mathrm{d} x .
\end{aligned}
$$

Testing $(1.1)_{2}$ by $|B|^{2} B$, taking the divergence-free property into account and using the Hölder inequality, we get

$$
\begin{aligned}
\frac{1}{4} & \frac{\mathrm{d}}{\mathrm{d} t} \int_{\mathbb{R}^{3}}|B|^{4} \mathrm{~d} x+\frac{1}{2}\|B \cdot \nabla B\|_{L^{2}}^{2}+\frac{1}{2}\left\|\nabla|B|^{2}\right\|_{L^{2}}^{2} \\
& =\int_{\mathbb{R}^{3}}(B \cdot \nabla) u \cdot|B|^{2} B \mathrm{~d} x+\int_{\mathbb{R}^{3}}(B \times(\nabla \times B)) \cdot \nabla \times\left(|B|^{2} B\right) \mathrm{d} x \\
& =-\int_{\mathbb{R}^{3}}(B \cdot \nabla)|B|^{2} B \cdot u \mathrm{~d} x+\int_{\mathbb{R}^{3}}(B \times(\nabla \times B))\left(\nabla|B|^{2} \times B\right) \mathrm{d} x \\
& \leq C \int_{\mathbb{R}^{3}}|u||B|^{3}|\nabla B| \mathrm{d} x+C \int_{\mathbb{R}^{3}}|B|^{3}|\nabla B|^{2} \mathrm{~d} x,
\end{aligned}
$$

where we used the fact $\|\nabla w\|_{L^{2}}=\|\nabla \times w\|_{L^{2}}$ if $\nabla \cdot w=0$.

Due to the following ones $(0 \leq r \leq 1)$

$$
\|w\|_{\dot{H}^{r}} \leq C\|w\|_{L^{2}}^{1-r}\|\nabla w\|_{L^{2}}^{r},
$$

it is easy to see that

$$
\begin{aligned}
& \frac{1}{4} \frac{\mathrm{d}}{\mathrm{d} t} \int_{\mathbb{R}^{3}}\left(|u|^{4}+|B|^{4}\right) \mathrm{d} x+\frac{1}{2}\left(\|u \cdot \nabla u\|_{L^{2}}^{2}+\|B \cdot \nabla B\|_{L^{2}}^{2}\right)+\frac{1}{2}\left(\left\|\nabla|u|^{2}\right\|_{L^{2}}^{2}+\left\|\nabla|B|^{2}\right\|_{L^{2}}^{2}\right) \\
& \quad \leq C\|\pi u\|_{L^{2}}\|u \cdot \nabla u\|_{L^{2}}+C\left\||B|^{2} u\right\|_{L^{2}}\|u \cdot \nabla u\|_{L^{2}}
\end{aligned}
$$




$$
\begin{aligned}
& +C\left\||u||B|^{2}\right\|_{L^{2}}\|B \cdot \nabla B\|_{L^{2}}+C\left\||\nabla B||B|^{2}\right\|_{L^{2}}\|B \cdot \nabla B\|_{L^{2}} \\
& \leq C\|\pi\|_{L^{4}}^{2}\|u\|_{L^{4}}^{2}+\frac{1}{2}\|u \cdot \nabla u\|_{L^{2}}^{2}+C\|u\|_{L^{4}}\left\||B|^{2}\right\|_{L^{4}}\|u \cdot \nabla u\|_{L^{2}} \\
& +C\|u\|_{L^{4}}\left\||B|^{2}\right\|_{L^{4}}\|B \cdot \nabla B\|_{L^{2}}+C\|\nabla B\|_{\dot{X}_{r}}\left\||B|^{2}\right\|_{L^{2}}^{1-r}\left\|\nabla|B|^{2}\right\|_{L^{2}}^{r}\|B \cdot \nabla B\|_{L^{2}} \\
& \leq C\|\pi\|_{\dot{B}_{\infty, \infty}^{-1}}\|\nabla \pi\|_{L^{2}}\|u\|_{L^{4}}^{2}+\frac{1}{2}\|u \cdot \nabla u\|_{L^{2}}^{2}+C\|u\|_{L^{4}}\|B\|_{L^{8}}^{2}\|u \cdot \nabla u\|_{L^{2}} \\
& +C\|u\|_{L^{4}}\|B\|_{L^{8}}^{2}\|B \cdot \nabla B\|_{L^{2}}+C\left(\|\nabla B\|_{X_{r}}^{\frac{2}{1-r}}\|B\|_{L^{4}}^{4}\right)^{\frac{1-r}{2}}\left(\left\|\nabla|B|^{2}\right\|_{L^{2}}^{2}\right)^{\frac{r}{2}}\left(\|B \cdot \nabla B\|_{L^{2}}^{2}\right)^{\frac{1}{2}} \\
& \leq C\|\pi\|_{\dot{B}_{\infty, \infty}^{-1}}^{2}\|u\|_{L^{4}}^{4}+\frac{1}{2}\|\nabla \pi\|_{L^{2}}^{2}+\frac{1}{2}\|u \cdot \nabla u\|_{L^{2}}^{2}+C\|u\|_{L^{4}}\|B\|_{L^{4}}\|B\|_{L^{\infty}}\|u \cdot \nabla u\|_{L^{2}} \\
& +C\|u\|_{L^{4}}\|B\|_{L^{4}}\|B\|_{L^{\infty}}\|B \cdot \nabla B\|_{L^{2}}+C\|\nabla B\|_{\dot{X}_{r}}^{\frac{2}{1-r}}\|B\|_{L^{4}}^{4}+\frac{1}{4}\left\|\nabla|B|^{2}\right\|_{L^{2}}^{2}+\frac{1}{4}\|B \cdot \nabla B\|_{L^{2}}^{2} \\
& \leq C\|\pi\|_{\dot{B}_{\infty, \infty}^{-1}}^{2}\|u\|_{L^{4}}^{4}+\frac{1}{2}\left(\|u \cdot \nabla u\|_{L^{2}}^{2}+\|B \cdot \nabla B\|_{L^{2}}^{2}\right)+\frac{1}{2}\|u \cdot \nabla u\|_{L^{2}}^{2} \\
& +C\|u\|_{L^{4}}^{2}\|B\|_{L^{4}}^{2}\|B\|_{L^{\infty}}^{2}+\frac{1}{2}\|u \cdot \nabla u\|_{L^{2}}^{2}+C\|u\|_{L^{4}}^{2}\|B\|_{L^{4}}^{2}\|B\|_{L^{\infty}}^{2} \\
& +\frac{1}{2}\|B \cdot \nabla B\|_{L^{2}}^{2}+C\|\nabla B\|_{\dot{X}_{r}}^{\frac{2}{1-r}}\|B\|_{L^{4}}^{4}+\frac{1}{4}\left\|\nabla|B|^{2}\right\|_{L^{2}}^{2}+\frac{1}{4}\|B \cdot \nabla B\|_{L^{2}}^{2} \\
& \leq \frac{1}{2}\|u \cdot \nabla u\|_{L^{2}}^{2}+\frac{1}{2}\|B \cdot \nabla B\|_{L^{2}}^{2}+\frac{1}{4}\left\|\nabla|B|^{2}\right\|_{L^{2}}^{2}+C\|\pi\|_{\dot{B}_{\infty, \infty}^{-1}}^{2}\|u\|_{L^{4}}^{4} \\
& +C\|B\|_{L^{\infty}}^{2}\left(\|u\|_{L^{4}}^{4}+\|B\|_{L^{4}}^{4}\right)+C\|\nabla B\|_{\dot{X}_{r}}^{\frac{2}{1-r}}\|B\|_{L^{4}}^{4} \\
& \leq \frac{1}{2}\|u \cdot \nabla u\|_{L^{2}}^{2}+\frac{1}{2}\|B \cdot \nabla B\|_{L^{2}}^{2}+\frac{1}{4}\left\|\nabla|B|^{2}\right\|_{L^{2}}^{2} \\
& +C\left(\|u\|_{L^{4}}^{4}+\|B\|_{L^{4}}^{4}\right)\left(\|\pi\|_{\dot{B}_{\infty, \infty}^{-1}}^{2}+\|B\|_{L^{\infty}}^{2}+\|\nabla B\|_{\dot{X}_{r}}^{\frac{2}{1-r}}\right),
\end{aligned}
$$

where we used the Young inequality. Consequently, we have

$$
\begin{aligned}
& \frac{1}{4} \frac{\mathrm{d}}{\mathrm{d} t} \int_{\mathbb{R}^{3}}\left(|u|^{4}+|B|^{4}\right) \mathrm{d} x+\frac{1}{2}\left(\|u \cdot \nabla u\|_{L^{2}}^{2}+\|B \cdot \nabla B\|_{L^{2}}^{2}\right)+\frac{1}{2}\left(\left\|\nabla|u|^{2}\right\|_{L^{2}}^{2}+\left\|\nabla|B|^{2}\right\|_{L^{2}}^{2}\right) \\
& \leq C\left(\|u\|_{L^{4}}^{4}+\|B\|_{L^{4}}^{4}\right)\left(\|\pi\|_{\dot{B}_{\infty, \infty}^{-1}}^{2}+\|B\|_{L^{\infty}}^{2}+\|\nabla B\|_{\dot{X}_{r}}^{\frac{2}{1-r}}\right) .
\end{aligned}
$$

By applying the standard Gronwall inequality, we obtain, for every $t \in[0, T]$,

$$
\begin{aligned}
& \|u\|_{L^{4}}^{4}+\|B\|_{L^{4}}^{4}+\int_{0}^{t}\left(\|u \cdot \nabla u(\tau)\|_{L^{2}}^{2}+\|B \cdot \nabla B(\tau)\|_{L^{2}}^{2}\right) \mathrm{d} \tau \\
& \quad+\int_{0}^{t}\left(\left\|\nabla|u|^{2}(\tau)\right\|_{L^{2}}^{2}+\left\|\nabla|B|^{2}(\tau)\right\|_{L^{2}}^{2}\right) \mathrm{d} \tau \\
& \leq C\left(\left\|u_{0}\right\|_{L^{2}}^{2}+\left\|B_{0}\right\|_{L^{2}}^{2}\right) \exp \left(\int_{0}^{T}\left(\|\pi(\tau)\|_{\dot{B}_{\infty, \infty}^{-1}}^{2}+\|B(\tau)\|_{L^{\infty}}^{2}+\|\nabla B(\tau)\|_{\dot{X}_{r}}^{\frac{2}{1-r}}\right) \mathrm{d} \tau\right) .
\end{aligned}
$$

This implies us that

$$
(u, B) \in L^{\infty}\left(0, T ; L^{4}\left(\mathbb{R}^{3}\right)\right) \subset L^{8}\left(0, T ; L^{4}\left(\mathbb{R}^{3}\right)\right)
$$


and

$$
(u . \nabla u, B . \nabla B) \in L^{2}\left(0, T ; L^{2}\left(\mathbb{R}^{3}\right)\right) .
$$

From (4.1) and the standard Serrin regularity criterion (see e.g., [12]), we have $(u, B)$ smooth on $(0, T) \times$ $\mathbb{R}^{3}$. Then, by using the standard arguments of the continuation of local solutions, it is easy to conclude that the above estimate (4.1) implies that the solution $(u(x, t), B(x, t))$ can be extended beyond $t=T$. This completes the proof by Theorem 2.7.

\section{Acknowledgments}

The part of the work was carried out, while the first author was long-term visitor at University of Catania. The hospitality and support of Catania University are graciously acknowledged. The authors are indebted to Professor Yong Zhou who kindly sent us the preprint [12]. The authors are also grateful to the anonymous referees for their constructive comments and helpful suggestions that have contributed to the final preparation of the paper.

Open Access. This article is distributed under the terms of the Creative Commons Attribution 4.0 International License (http://creativecommons.org/licenses/by/4.0/), which permits unrestricted use, distribution, and reproduction in any medium, provided you give appropriate credit to the original author(s) and the source, provide a link to the Creative Commons license, and indicate if changes were made.

\section{References}

1. Acheritogaray, M., Degond, P., Frouvelle, A., Liu, J.G.: Kinetic formulation and global existence for the Hallmagnetohydrodynamics system. Kinet. Relat. Models 4, 901-918 (2011)

2. Chae, D., Lee, J.: On the blow-up criterion and small data global existence for the Hall-magnetohydrodynamics. J. Differ. Equ. 256(11), 3835-3858 (2014)

3. Chae, D., Schonbek, M.: On the temporal decay for the Hall-magnetohydrodynamic equations. J. Differ. Equ. 255(11), 3971-3982 (2013)

4. Chae, D., Weng, S.: Singularity formation for the incompressible Hall-MHD equations without resistivity, 19 December 2013. arXiv:1312.5519v1[math. AP]

5. Chae, D., Wu, J.: Local well-posedness for the Hall-MHD equations with fractional magnetic diffusion. arXiv:1404.0486v1[math. AP] 2 Apr (2014)

6. Chae, D., Degond, P., Liu, J.G.: Well-posedness for Hall-magnetohydrodynamics. Ann. Inst. H. Poincaré Anal. Non Linéaire 31, 555-565 (2014)

7. Dumas, E., Sueur, F.: On the weak solutions to the Maxwell-Landau-Lifshitz equations and to the Hall magnetohydrodynamic equations. Commun. Math. Phys. 330, 1179-1225 (2014)

8. Dreher, J., Runban, V., Grauer, R.: Axisymmetric flows in Hall-MHD: a tendency towards finite-time singularity formation. Phys. Scr. 72, 451-455 (2005)

9. Fan, J., Ozawa, T.: Regularity criteria for Hall-magnetohydrodynamics and the space-time monopole equation in Lorenz gauge. Contemp. Math. 612, 81-89 (2014)

10. Fan, J., Ozawa, T.: Regularity criteria for the density-dependent Hall-magnetohydrodynamics. Appl. Math. Lett. 36, 14$18(2014)$

11. Fan, J., Huang, S., Nakamura, G.: Well-posedness for the axisymmetric incompressible viscous Hallmagnetohydrodynamic equations. Appl. Math. Lett. 26(9), 963-967 (2013)

12. Fan, J., Jia, X., Nakamura, G., Zhou, Y.: On well-posedness and blowup criteria for the magnetohydrodynamics with the Hall and ion-slip effects. Z. Angew. Math. Phys. 66(4), 1695-1706 (2015)

13. Fan, J., Li, F., Nakamura, G., Tan, Z.: Regularity criteria for the three-dimensional magnetohydrodynamic equations. J. Differ. Equ. 256, 2858-2875 (2014)

14. Fan, J., Li, F., Nakamura, G.: Regularity criteria for the incompressible Hall-magnetohydrodynamic equations. Nonlinear Anal. TMA 109, 173-179 (2014)

15. Forbes, T.G.: Magnetic reconnection in solar flares. Geophys. Astrophys. Fluid Dyn. 62, 15-36 (1991)

16. Gala, S., Lemarié-Rieusset, P.G.: Multipliers between Sobolev spaces and fractional differentiation. J. Math. Anal. Appl. 322, 1030-1054 (2006) 
17. Galdi, G.P., Padula, R.: A new approach to energy theory in the stability of fluid motion. Arch. Rat. Mech. Anal. 110, 187-286 (1990)

18. Meyer, Y., Gerard, P., Oru, F.: Inégalités de Sobolev précisées, Séminaire Équations aux dérivées partielles (Polytechnique) (1996-1997), Exp. No. 4, p. 8

19. Mulone, G., Solonnikov, V.A.: On an initial boundary-value problem for the equation of magnetohydrodynamics with the Hall and ion-slip effects. J. Math. Sci. 87, 3381-3392 (1997)

20. Maiellaro, M.: Uniqueness of MHD thermodiffusive mixture flows with Hall and ion-slip effects. Meccanica 12, 9-14 (1977)

21. Mulone, G., Salemi, F.: Some continuous dependence theorems in MHD with Hall and ion-slip currents in unbounded domains. Rend. Ac. Sci. Fis. Mat. Napoli. 55, 139-152 (1988)

22. Wu, H.: Strong solution to the incompressible MHD equations with vacuum. Comput. Math. Appl. 61, 2742-2753 (2011)

23. Simakov, A.N., Chacón, L.: Quantitative, analytical model for magnetic reconnection in Hall magnetohydrodynamics. Phys. Rev. Lett. 101, 105003 (2008)

24. Triebel, H.: Interpolation Theory, Function Spaces, Differential Operators, North-Holland Mathematical Library 18. North-Holland, Amsterdam-New York (1978)

25. Zhou, Y., Fan, J.: A regularity criterion for the density-dependent magnetohydrodynamic equations. Math. Methods Appl. Sci. 33, 1350-1355 (2010)

26. Zhou, Y., Gala, S.: Regularity criteria for the solutions to the 3D MHD equations in the multiplier space, Z. Angew. Math. Phys. 61, 193-199 (2010)

Sadek Gala

Present address:

Department of Mathematics

University of Mostaganem

Box 227, Mostaganem, Algeria

e-mail: gala.sadek@gmail.com

Sadek Gala and Maria Alessandra Ragusa

Dipartimento di Mathematica e Informatica

Università di Catania

Viale Andrea Doria, 6

95125 Catania, Italy

e-mail: maragusa@dmi.unict.it

(Received: March 27, 2015; revised: December 28, 2015) 